\title{
Patterns and determinants of treatment for coronary artery disease
}

\section{A cross-sectional study in the Kingdom of Saudi Arabia}

Sameer H. Al-Ghamdi, MBBS, MD, Khalid Hadi Aldosari, MBBS, Mansour M. AlAjmi, MBBS, MD.

\begin{abstract}
الأهداف: تحديد الخصائص السريرية وخصائص تصوير الأوعية التاجية

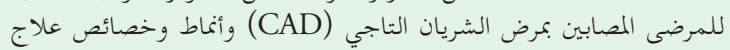

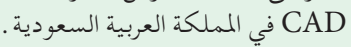

المنهجية : أجرينا دراسة مقطعية شملت 242 مريضًا في ثلاثة مستشفيات

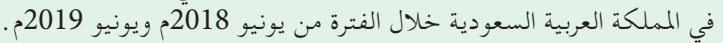

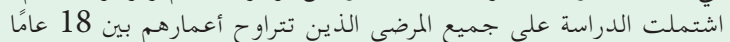

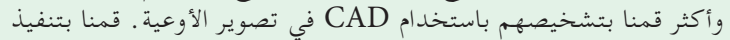

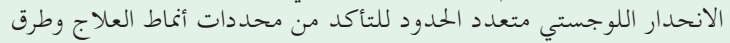

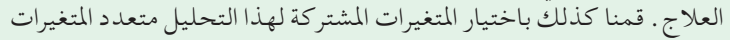
بناءً على الانحدار قدات أحادية المتغير.

النتائج: بلغ متوسط \pm الانحراف المعياري لمجتمع الدراسة 58.3 ـ 58.8 ـ 11.8

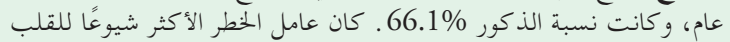

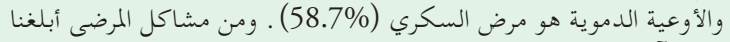
عن الآفات التي تنطوي على مرضى الشريان الأمامي الأيسر النازل

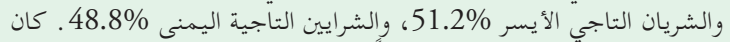

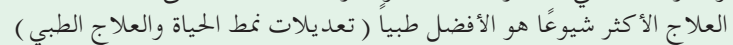

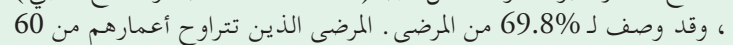

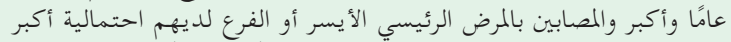

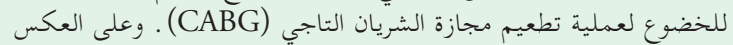

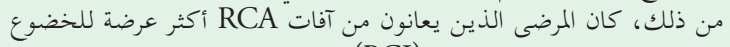

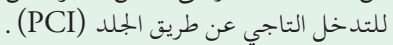

الحملاصة : كان عمر المريض وموقع التشريح لآفات تصلب الشرايين التاجية

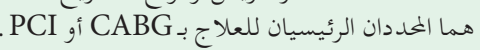

Objectives: To determine the clinical and coronary angiographic characteristics of patients with coronary artery disease $(\mathrm{CAD})$ and the patterns and determinants of CAD treatment in Kingdom of Saudi Arabia (KSA).

Methods: We conducted a cross-sectional study including 242 patients at 3 hospitals in KSA between June 2018 and June 2019. We included all patients aged $\geq 18$ years diagnosed with CAD on angiography. We carried out a multinomial logistic regression to ascertain the determinants of treatment patterns and treatment modalities. Covariates for this multivariate analysis were selected based on univariate regressions.

Results: The study population had a mean \pm standard deviation of $58.3 \pm 11.8$ years, and $66.1 \%$ were male. The most frequent cardiovascular risk factor was diabetes $(58.7 \%)$. Lesions involving the left anterior descending were reported among $68.6 \%$, left circumflex among $51.2 \%$, and right coronary arteries (RCA) among $48.8 \%$ of our patients. The most common treatment was the best medical therapy (lifestyle modifications and medical management), prescribed to $69.8 \%$ of patients. Patients aged $\leq 60$ years with the left main disease or disease of the ramus had a higher likelihood of undergoing coronary artery bypass grafting (CABG). Contrarily, patients with RCA lesions were more likely to undergo a percutaneous coronary intervention (PCI).

Conclusion: Patient age and anatomical localization of coronary atherosclerotic lesions were the main determinants of treatment with CABG or PCI.

Keywords: coronary artery disease, coronary artery bypass graft, percutaneous coronary intervention, Saudi Arabia

Saudi Med J 2021; Vol. 42 (8): 895-902

doi: 10.15537/smj.2021.42.8.20210219

From the Department of Family and Community Medicine (Al-Ghamdi, AlAjmi); from the College of Medicine (Aldosari), Prince Sattam Bin Abdulaziz University, Al Kharj; and from the Adult Critical Care Medicine Department (Aldosari), Security Forces Hospital Program, Riyadh, Kingdom of Saudi Arabia.

Received 17th March 2021. Accepted 27th June 2021.

Address correspondence and reprint request to: Dr. Sameer H. AlGhamdi, Department of Family and Community Medicine, College of Medicine. Prince Sattam Bin Abdulaziz University, Al Kharj, Kingdom of Saudi Arabia.E-mail: sh.alghamdi@psau.edu.sa ORCID ID: https://orcid.org/0000-0003-0506-0574 
Tn 2019, coronary artery disease (CAD) was the foremost reason for disability and mortality worldwide, responsible for 182 million disabilityadjusted life years and nearly 9.1 million deaths. ${ }^{1}$ In the Kingdom of Saudi Arabia (KSA), a literature review of CAD risk factors delineates that almost a quarter of all adults have hypertension and diabetes while more than half have hypercholesterolemia. ${ }^{2-4}$ Fueled by such increasingly prevalent patient factors in the KSA, the risk and subsequently the burden of cardiovascular disease (CVD) has considerably risen. The Global Burden of Disease reported that CAD was the foremost cause of mortality in the country in 2019, responsible for $23.1 \%$ of all deaths. ${ }^{1}$ Despite the high burden of CAD in the $\mathrm{KSA}$, there is limited data on the epidemiology of the disease in the country, especially its management.

Patients with CAD are usually managed with medical therapy alone or revascularization procedures, such as coronary artery bypass grafting (CABG) or percutaneous coronary intervention (PCI). The choice between treatment modalities is complex, which depends on patient factors, preferences, and invasive coronary angiographic findings. While the American Heart Association and the American College of Cardiology recommend different treatment modalities based on the type of coronary syndromes and the anatomical localization as determined by invasive coronary angiography, ${ }^{5}$ these recommendations are based on studies conducted in the Western world and may not be readily applicable to the Saudi population.

In general, revascularization is preferred over medical therapy in patients with acute coronary syndromes, symptomatic CAD despite maximal medical treatment, those unable to tolerate medical therapy, or those with angiographic findings suggestive of substantial luminal narrowing. ${ }^{6}$ Coronary artery bypass grafting is considered the gold standard therapeutic procedure in patients suffering from a 3-vessel disease or left main coronary artery involvement. ${ }^{7}$ It is also preferred in cases of single- or double-vessel disease wherein PCI cannot achieve complete revascularization. Percutaneous coronary intervention is usually the preferred modality of treatment in single-vessel afflictions besides specific types of double-vessel afflictions. It is also beneficial

Disclosure. This study was funded by the Deanship of Scientific Research, Prince Sattam Bin Abdulaziz University, Al Kharj, Kingdom of Saudi Arabia, Project No. 2020/03/16884. in enhancing patient quality of life in those who are not fit for or refuse CABG. The final decision is often made based on the doctors' discretion and tailored to the individual patient. ${ }^{6}$

There is little evidence regarding CAD management post invasive coronary angiography specific to the Saudi population. ${ }^{5}$ Such data will enable Saudi cardiologists to follow a more population-specific evidence-based approach towards treating CAD. Hence, this study's objectives were to determine the clinical and coronary angiographic characteristics of patients with CAD and the patterns and determinants of CAD treatment in 3 major hospitals in KSA.

Methods. We conducted a multi-centric crosssectional study between June 2018 and 2019 at King Khalid Hospital \& Prince Sultan Centre for Healthcare, Al-Kharj; the Cardiovascular Medicine and Cardiovascular Surgery Departments, King Khalid Hospital, Riyadh; and Cardiology Department, Military Industries Corporation Hospital, Al-Kharj, KSA. We identified keywords regarding CAD epidemiology and treatment and performed a comprehensive literature review on PubMed and GoogleScholar databases to find prior related research to guide the design and write-up of this research project. Our study obtained ethical approval by the Institutional Review Board of Prince Sattam Bin Abdulaziz University and was conducted according to the principles of the Helsinki Declaration. Prior to enrollment into the study, all participants provided written informed consent.

Convenient sampling was used to recruit patients between June 2018 and June 2019. Patients who presented to the study sites during the recruitment period were eligible if they were aged $\geq 18$ years, current residents of the KSA, and diagnosed with CAD based on at least one identifiable coronary lesion on a coronary angiogram. Patients diagnosed with $\mathrm{CAD}$ via other non-invasive coronary artery imaging modalities and those whose electronic medical records had incomplete or missing data points were excluded.

We collected details on age, gender, and risk factors that pertain to CVDs, such as diabetes status, smoking history, hypertension, dyslipidemia, and any other comorbidities. Information from the coronary angiogram included indications such as ST-elevation myocardial infarction (STEMI), non-ST-elevation myocardial infarction (NSTEMI), atypical angina, and stable or unstable angina and the localization, number, and severity of coronary atherosclerotic plaques. We defined coronary artery stenosis as $\geq 50 \%$ diameter stenosis of the left main coronary artery or 
$\geq 70 \%$ diameter stenosis of at least one of the major epicardial coronary arteries. ${ }^{8}$ We gathered information on the treatment modalities, which included the best medical therapy (BMT) and surgical procedures, such as PCI and CABG. We defined BMT as the use of antianginal drugs (nitrate or $\beta$-blocker or calcium channel blocker) and statins in all patients, with an additional angiotensin-converting enzyme inhibitor or an angiotensin receptor blocker only in patients with either a left ventricular dysfunction $(<50 \%)$, type 2 diabetes, chronic renal disease, hypertension, peripheral artery disease, or a history of a cerebrovascular accident. Best medical therapy was systematically associated with lifestyle modifications targeting weight loss, diet, and physical activity. ${ }^{9}$

Statistical analysis. Data were entered into and analyzed by IBM SPSS Statistics for Windows version 23.0 (IBMCorp, Armonk, NY, USA). As our continuous variables were normally distributed, we reported them as mean \pm standard deviation (SD). Categorical variables are reported as frequencies and percentages. We constructed a multinomial logistic regression model to explore patient clinical and coronary angiographic characteristics that may potentially have an association with surgical procedures such as PCI and CABG. We did so by considering BMT as the reference category. The multinomial logistic regression was conducted in 2 steps: first, a univariate regression to identify statistically significant factors and second, a multivariate logistic regression for adjusted analysis. In the univariate analysis, all individual variables/factors were evaluated based on a conventional significance level $<0.25$. The multivariate analysis model was generated considering all variables deemed statistically significant by univariate analysis. All analyses were 2-tailed, set at $95 \%$ confidence intervals (CIs), with $p$-values of $<0.05$ deemed statistically significant.

Results. The study population consisted of 242 patients having a mean \pm SD age of $58.3 \pm 11.8$ years, and $66.1 \%$ were men. The most frequent cardiovascular (CV) risk factors were diabetes $(58.7 \%)$, hypertension (55.4\%), dyslipidemia (30.6\%), and current smoking (28.1\%). History of CAD was reported in $15.7 \%$ of patients (Table 1). Most patients presented with acute coronary syndromes (59.8\%), 32.2\% had unstable angina, $19.8 \%$ STEMI, and $17.8 \%$ NSTEMI.

Among our patients, $68.6 \%$ had a left anterior descending artery (LAD) lesion, 51.2\% right coronary artery (RCA) lesion, and 48.8\% left circumflex artery (LCX) lesion. Multivessel disease was found in $57.4 \%$ of patients. In terms of coronary vessel dominance, $57.5 \%$

\begin{tabular}{|c|c|c|}
\hline Characteristics & $\mathbf{n}$ & $(\%)$ \\
\hline Age (years) mean $\pm \mathrm{SD}$ & \multicolumn{2}{|c|}{$58.3 \pm 11.83$} \\
\hline$\leq 60$ & 138 & $(57.0)$ \\
\hline$>60$ & 104 & $(43.0)$ \\
\hline \multicolumn{3}{|l|}{ Gender } \\
\hline Female & 82 & (33.9) \\
\hline Male & 160 & $(66.1)$ \\
\hline \multicolumn{3}{|l|}{ Smoking status } \\
\hline Ex-smoker & 15 & $(6.2)$ \\
\hline Non-smoker & 159 & $(65.7)$ \\
\hline Smoker & 68 & $(28.1)$ \\
\hline \multicolumn{3}{|l|}{ Indications } \\
\hline Atypical angina & 67 & $(27.7)$ \\
\hline NSTEMI & 43 & $(17.8)$ \\
\hline Stable angina & 6 & $(2.5)$ \\
\hline STEMI & 48 & $(19.8)$ \\
\hline Unstable angina & 78 & $(32.2)$ \\
\hline \multicolumn{3}{|l|}{ Comorbidities* } \\
\hline Diabetes mellitus & 142 & $(58.7)$ \\
\hline Hypertension & 134 & $(55.4)$ \\
\hline Dyslipidemia & 73 & $(30.2)$ \\
\hline History of heart failure & 35 & $(14.5)$ \\
\hline History of ACS & 31 & $(12.8)$ \\
\hline Hypothyroidism & 16 & $(6.6)$ \\
\hline Renal disease & 12 & $(5.0)$ \\
\hline History of IHD & 38 & $(15.7)$ \\
\hline Severe aortic stenosis & 11 & $(4.5)$ \\
\hline Rheumatic heart disease & 11 & $(4.5)$ \\
\hline Others & 12 & $(4.9)$ \\
\hline \multicolumn{3}{|l|}{ Number of comorbidities } \\
\hline No comorbidities & 59 & $(24.4)$ \\
\hline Single comorbidity & 39 & $(16.1)$ \\
\hline 2 comorbidities & 71 & $(29.3)$ \\
\hline Multiple comorbidities & 73 & $(30.2)$ \\
\hline \multicolumn{3}{|c|}{$\begin{array}{l}{ }^{*} \text { Categories within this variable are not mutually } \\
\text { exclusive. NSTEMI: non-ST-elevation myocardial } \\
\text { infarction, STEMI: ST-elevation myocardial } \\
\text { infarction, ACS: acute coronary syndrome, IHD: } \\
\text { ischemic heart disease }\end{array}$} \\
\hline
\end{tabular}

of all patients had RCA dominance, whereas $32.6 \%$ of them had left coronary artery dominance (Table 2).

The management was conservative in most cases, with $69.8 \%$ who received BMT exclusively. Only $11.6 \%$ of patients underwent PCI, and $18.6 \%$ were managed with CABG (Table 3).

In univariable regression analysis, considering BMT as the reference treatment modality, factors associated with CABG were lesions in the left main artery, LAD, ramus, $\mathrm{LCX}, \mathrm{RCA}$, and multivessel diseases. In the multivariable analysis, patients $<60$ years of age (adjusted odds ratio $[\mathrm{aOR}]$ : 4.30, CI: 1.6-11.8, $p=0.005)$, 
Table 2 - Distribution of pattern of artery lesions $(\mathrm{N}=242)$.

\begin{tabular}{lcc}
\hline Pattern of artery lesions & $\mathbf{n}$ & $(\%)$ \\
\hline $\begin{array}{l}\text { Pattern of artery lesions } \\
\text { LM lesion }\end{array}$ & 23 & $9(.5)$ \\
LAD lesion & 166 & $(68.6)$ \\
Ramus lesion & 19 & $(7.9)$ \\
LCX lesion & 118 & $(48.8)$ \\
RCA lesion & 124 & $(51.2)$ \\
Multi vessel disease & & \\
Yes & 139 & $(57.4)$ \\
No & 103 & $(42.6)$ \\
Dominant artery & \\
Both & 23 & $(9.5)$ \\
Left & 79 & $(32.6)$ \\
Right & 140 & $(57.9)$ \\
\hline & $*$ Categories within variable are not mutually \\
exclusive. LM: left main artery lesion, LAD: \\
left anterior descending artery lesion, LCX: left \\
circumflex artery lesion, RCA: right coronary \\
$\quad$ artery lesion \\
$\quad$
\end{tabular}

with disease of the ramus (aOR: 6.90, CI: 1.8-26.8; $p=0.005$ ), or the left main artery (aOR: $4.50, \mathrm{CI}$ : $1.5-16.6, p=0.01)$ were more likely to undergo CABG (Table 4). Factors associated with PCI in the univariable analysis included age $\leq 60$ years, female gender, diabetes, hypertension, dyslipidemia, and lesion in the RCA. In the multivariable analysis, patients with a lesion in the RCA were more likely to undergo PCI (aOR: 14.6, CI: 2.3-93.1); $p=0.005$ ) as compared to those with a lesion in the LAD (aOR: 0.09, CI: 0.01-0.79) (Table 5).

Discussion. This study aimed to evaluate the patterns and determinants of different treatment modalities for CAD in 3 major hospitals in the KSA. The study revealed that diabetes, hypertension, dyslipidemia, and smoking are highly prevalent in patients with CAD in our settings. The LAD artery lesion was the most common lesion. The treatment of CAD was mostly conservative, with more than two-thirds of patients receiving only medical therapy. Age and the anatomical localization of the CAD lesions were the main factors associated with treatment with CABG or PCI. Most often the lesions of the ramus and the left main artery required CABG while the lesion of RCA required PCI.

Irrespective of age, the incidence of $\mathrm{CAD}$ is higher in men compared to women. ${ }^{10,11}$ Although STEMI is more common in men, women tend to have a worse prognosis after myocardial infarction. ${ }^{12}$ Microvascular angina is more common in women. ${ }^{12}$ It has been reported that CVDs account for $40 \%$ and $49 \%$ of all
Table 3 - Distribution and association management modalities with associated factors $(\mathrm{N}=242)$.

\begin{tabular}{|c|c|c|c|}
\hline \multirow[b]{2}{*}{ Associated factors } & \multicolumn{3}{|c|}{ Management modalities } \\
\hline & $\begin{array}{l}\text { Best medical } \\
\text { therapy }\end{array}$ & CABG & PCI \\
\hline \multicolumn{4}{|l|}{ Age (years) } \\
\hline$\leq 60$ & $105(76.1)$ & $23(16.7)$ & $10(7.2)$ \\
\hline$>60$ & $64(61.5)$ & $22(2.2)$ & $18(17.3)$ \\
\hline \multicolumn{4}{|l|}{ Gender } \\
\hline Male & $117(73.1)$ & $32(20)$ & $11(6.9)$ \\
\hline Female & $52(63.4)$ & $13(15.9)$ & $17(20.7)$ \\
\hline \multicolumn{4}{|l|}{ Smoking status } \\
\hline Current/ex-smoker & $56(67.9)$ & $20(24.7)$ & $6(7.4)$ \\
\hline Non-smoker & $113(71.1)$ & $25(15.7)$ & $21(13.2)$ \\
\hline \multicolumn{4}{|l|}{ Diabetes mellitus } \\
\hline Yes & $91(64.1)$ & $26(18.3)$ & $25(17.6)$ \\
\hline No & $78(78.0)$ & $19(19.0)$ & $3(3.0)$ \\
\hline \multicolumn{4}{|l|}{ Hypertension } \\
\hline Yes & $89(66.4)$ & $23(17.2)$ & $22(16.4)$. \\
\hline No & $80(74.1)$ & $22(20.4)$ & $6(5.6)$ \\
\hline \multicolumn{4}{|l|}{ Dyslipidemia } \\
\hline Yes & $45(61.6)$ & $12(16.4)$ & $16(21.9)$ \\
\hline No & $124(73.4)$ & $33(19.5)$ & $12(7.1)$ \\
\hline \multicolumn{4}{|l|}{ Diagnosis } \\
\hline Atypical angina & $48(71.6)$ & $16(23.9)$ & $3(4.5)$ \\
\hline Stable angina & $5(83.3)$ & $1(16.7)$ & $0(0.0)$ \\
\hline Unstable angina & $52(66.7)$ & $10(12.8)$ & $16(20.5)$ \\
\hline NSTEMI & $29(67.4)$ & $11(25.6)$ & $3(7.0)$ \\
\hline STEMI & $35(72.9)$ & $7(14.6)$ & $6(12.5)$ \\
\hline \multicolumn{4}{|l|}{ LM lesion } \\
\hline Yes & $12(52.2)$ & $11(47.8)$ & $0(0.0)$ \\
\hline No & $157(71.7)$ & $34(15.5)$ & $28(12.8)$ \\
\hline \multicolumn{4}{|l|}{$L A D$ lesion } \\
\hline Yes & $116(69.9)$ & $43(25.9)$ & $7(4.2)$ \\
\hline No & $53(69.7)$ & $2(2.6)$ & $21(27.6)$ \\
\hline \multicolumn{4}{|l|}{ Ramus } \\
\hline Yes & $6(31.6)$ & $13(68.4)$ & $0(0.0)$ \\
\hline No & $163(73.1)$ & $32(14.3)$ & $28(12.6)$ \\
\hline \multicolumn{4}{|l|}{$L C X$} \\
\hline Yes & $73(61.9)$ & $37(31.4)$ & $8(6.8)$ \\
\hline No & $96(77.4)$ & $8(6.5)$ & $20(16.1)$ \\
\hline \multicolumn{4}{|l|}{$R C A$} \\
\hline Yes & $65(52.4)$ & $35(28.2)$ & $24(19.4)$ \\
\hline No & $104(88.1)$ & $10(8.5)$ & $4(3.4)$ \\
\hline \multicolumn{4}{|l|}{ Dominant artery } \\
\hline Right & $79(56.4)$ & $39(27.9)$ & $22(15.7)$ \\
\hline Left & $73(92.4)$ & $4(5.1)$ & $2(2.5)$ \\
\hline Both & $17(73.9)$ & $2(8.7)$ & $4(17.4)$ \\
\hline \multicolumn{4}{|l|}{$M V D$} \\
\hline Yes & $85(61.2)$ & $44(31.7)$ & $10(7.2)$ \\
\hline No & $84(81.6)$ & $1(1.0)$ & $18(17.4)$ \\
\hline Total & $169(69.8)$ & $45(18.6)$ & $28(11.6)$ \\
\hline \multicolumn{4}{|c|}{$\begin{array}{l}\text { Values are presented as number and percentages (\%). BMT: best medical } \\
\text { therapy, PCI: percutaneous coronary intervention, CABG: coronary } \\
\text { artery bypass grafting, NSTEMI: non-ST-elevation myocardial infarction, } \\
\text { STEMI: ST-elevation myocardial infarction, LM: left main lesion, } \\
\text { RCA: right coronary arteries, LAD: left anterior descending artery, } \\
\text { LCX: left circumflex artery, MVD: coronary microvascular disease }\end{array}$} \\
\hline
\end{tabular}


Table 4 - Association between associated factors with management modalities (univariate regression analysis).

\begin{tabular}{|c|c|c|c|}
\hline \multirow{2}{*}{ Associated factors } & \multicolumn{3}{|c|}{$\begin{array}{c}\text { Univariate regression analysis } \\
\text { Unadjusted OR ( } 95 \% \mathrm{CI} ; P \text {-value) }\end{array}$} \\
\hline & $\begin{array}{l}\text { Best medical } \\
\text { therapy }\end{array}$ & CABG & PCI \\
\hline \multicolumn{4}{|l|}{ Age (years) } \\
\hline$\leq 60$ & Ref. & $1.57(0.81-3.04) ; p=0.182^{*}$ & $2.95(1.28-6.79) ; p=0.011^{*}$ \\
\hline$>60$ & Ref. & 1 & 1 \\
\hline \multicolumn{4}{|l|}{ Gender } \\
\hline Male & Ref. & $1.09(0.53-2.25) ; p=0.807$ & $0.29(0.13-0.66) ; p=0.003^{*}$ \\
\hline Female & Ref. & 1 & 1 \\
\hline \multicolumn{4}{|l|}{ Smoking status } \\
\hline $\begin{array}{l}\text { Current/ex- } \\
\text { smoker }\end{array}$ & Ref. & $1.64(0.84-3.21) ; p=0.146^{*}$ & $0.59(0.22-1.54) ; p=0.278$ \\
\hline Non-smoker & Ref. & 1 & 1 \\
\hline \multicolumn{4}{|l|}{ Diabetes mellitus } \\
\hline Yes & Ref. & $1.17(0.60-2.28) ; p=0.638$ & $7.14(2.08-24.56) ; p=0.002^{*}$ \\
\hline No & Ref. & 1 & 1 \\
\hline \multicolumn{4}{|l|}{ Hypertension } \\
\hline Yes & Ref. & $0.94(0.49-1.81) ; p=0.853$ & $3.29(1.27-8.54) ; p=0.014^{*}$ \\
\hline No & Ref. & 1 & 1 \\
\hline \multicolumn{4}{|l|}{ Dyslipidemia } \\
\hline Yes & Ref. & $1.0(0.48-2.11) ; p=0.996$ & $3.67(1.61-8.36) ; p=0.002^{*}$ \\
\hline No & Ref. & 1 & 1 \\
\hline \multicolumn{4}{|l|}{ Diagnosis } \\
\hline Atypical angina & Ref. & $1.67(0.62-4.48) ; p=0.312$ & $0.37(0.09-1.56) ; p=0.173^{*}$ \\
\hline Stable angina & Ref. & $1.0(0.10-9.93) ; p=1.000$ & -ב--- \\
\hline Unstable angina & Ref. & $0.96(0.33-2.77) ; p=0.942$ & $1.79(0.64-5.04) ; p=0.266$ \\
\hline NSTEMI & Ref. & $1.89(0.65-5.52) ; p=0.240^{*}$ & $0.60(0.14-2.63) ; p=0.50$ \\
\hline STEMI & Ref. & 1 & 1 \\
\hline \multicolumn{4}{|l|}{ LM lesion } \\
\hline Yes & Ref. & $4.23(1.72-10.4) ; p=0.002^{*}$ & ------ \\
\hline No & Ref. & 1 & ------ \\
\hline \multicolumn{4}{|l|}{ LAD lesion } \\
\hline Yes & Ref. & $9.82(2.29-42.07) ; p=0.002^{*}$ & $0.15(0.06-42.07) ; p=0.000^{*}$ \\
\hline No & Ref. & 1 & 1 \\
\hline \multicolumn{4}{|l|}{ Ramus } \\
\hline Yes & Ref. & $11.04(3.91-31.19) ; p=0.002^{*}$ & ------ \\
\hline No & Ref. & 1 & 1 \\
\hline \multicolumn{4}{|l|}{$L C X$} \\
\hline Yes & Ref. & $6.08(2.67-13.85) ; p=0.000^{*}$ & $0.53(0.22-1.26) ; p=0.15^{*}$ \\
\hline No & Ref. & 1 & 1 \\
\hline \multicolumn{4}{|l|}{$R C A$} \\
\hline Yes & Ref. & 5.6(2.59-12.07); $p=0.000^{*}$ & 9.60 (3.19-28.93); $p=0.000^{*}$ \\
\hline No & Ref. & 1 & 1 \\
\hline \multicolumn{4}{|l|}{ Dominant artery } \\
\hline Right & Ref. & $4.19(0.92-19.1) ; p=0.063^{*}$ & $1.18(0.36-3.88) ; p=0.781$ \\
\hline Left & Ref. & $0.47(0.08-2.76) ; p=0.400$ & $0.12(0.02-0.69) ; p=0.018^{*}$ \\
\hline Both & Ref. & 1 & 1 \\
\hline \multicolumn{4}{|l|}{$M V D$} \\
\hline Yes & Ref. & $43.48(5.86-322.88) ; p=0.000^{*}$ & $0.55(0.24-1.26) ; p=0.157^{*}$ \\
\hline No & Ref. & 1 & 1 \\
\hline
\end{tabular}

Significance levels: for unadjusted/univariate analysis $<0.25$, for multivariate analysis $<0.05$.

Multinomial logistic regression used. The reference category is: "best medical therapy". OR: odds ratio ( $95 \%$ confidence interval). PCI: percutaneous coronary intervention, CABG: coronary artery bypass grafting, NSTEMI: non-ST-elevation myocardial infarction, STEMI: ST-elevation myocardial infarction, LM: left main lesion, RCA: right coronary arteries, LAD: left anterior descending artery, LCX: left circumflex artery, MVD: coronary microvascular disease, *statistically significance value 
Patterns and determinants of treatment for CAD ... Al-Ghamdi et al

Table 5 - Association between associated factors with management modalities (multivariate regression analysis).

\begin{tabular}{|c|c|c|c|}
\hline \multirow[t]{2}{*}{ Associated factors } & \multicolumn{3}{|c|}{$\begin{array}{l}\text { Multivariate regression analysis } \\
\text { Adjusted OR ( } 95 \% \text { CI; } P \text {-value) }\end{array}$} \\
\hline & Best medical therapy & CABG & PCI \\
\hline \multicolumn{4}{|l|}{ Age groups } \\
\hline$\leq 60$ years & Ref. & $4.27(1.55-11.8) ; p=0.005^{*}$ & $1.26(0.33-4.89) ; p: 0.738$ \\
\hline$>60$ years & Ref. & 1 & 1 \\
\hline \multicolumn{4}{|l|}{ Gender } \\
\hline Male & Ref. & ----- & $0.31(0.07-1.39) ; p: 0.126$ \\
\hline Female & Ref. & ----- & 1 \\
\hline \multicolumn{4}{|l|}{ Smoking status } \\
\hline Current/ex-smoker & Ref. & $0.98(0.33-2.87) ; p=0.965$ & ----- \\
\hline Non-smoker & Ref. & 1 & ------ \\
\hline \multicolumn{4}{|l|}{ Diabetes mellitus } \\
\hline Yes & Ref. & ------ & $4.07(0.81-20.4) ; p=0.089$ \\
\hline No & Ref. & ------ & 1 \\
\hline \multicolumn{4}{|l|}{ Hypertension } \\
\hline Yes & Ref. & ------ & $0.85(0.19-3.68) ; p=0.824$ \\
\hline No & Ref. & ----- & 1 \\
\hline \multicolumn{4}{|l|}{ Dyslipidemia } \\
\hline Yes & Ref. & ------ & $1.42(0.29-6.99) ; p=0.670$ \\
\hline No & Ref. & ------ & 1 \\
\hline \multicolumn{4}{|l|}{ Diagnosis } \\
\hline Atypical angina & Ref. & $1.63(0.41-6.63) ; p=0.490$ & $1.14(0.14-9.24) ; p=0.905$ \\
\hline Stable angina & Ref. & $1.57(0.09-24.73) ; p=0.751$ & ----- \\
\hline Unstable angina & Ref. & $1.23(0.35-4.29) ; p=0.747$ & $0.72(0.10-5.22) ; p=0.748$ \\
\hline NSTEMI & Ref. & $0.894(0.21-3.89) ; p=0.881$ & $0.72(0.09-5.93) ; p=0.761$ \\
\hline STEMI & Ref. & 1 & 1 \\
\hline \multicolumn{4}{|l|}{ LM lesion } \\
\hline Yes & Ref. & $4.94(1.47-16.61) ; p=0.010^{*}$ & ----- \\
\hline No & Ref. & 1 & ------ \\
\hline \multicolumn{4}{|l|}{ LAD lesion } \\
\hline Yes & Ref. & $0.83(0.08-8.75) ; p=0.875$ & $0.088(0.01-0.79) ; p=0.030^{*}$ \\
\hline No & Ref. & 1 & 1 \\
\hline \multicolumn{4}{|l|}{ Ramus } \\
\hline Yes & Ref. & $6.88(1.77-26.79) ; p=0.005^{*}$ & ------ \\
\hline No & Ref. & 1 & ----- \\
\hline \multicolumn{4}{|l|}{$L C X$} \\
\hline Yes & Ref. & $1.71(0.46-6.35) ; p=0.422$ & $1.36(0.22-8.25) ; p=0.739$ \\
\hline No & Ref. & 1 & 1 \\
\hline \multicolumn{4}{|l|}{$R C A$} \\
\hline Yes & Ref. & $1.64(0.59-4.55) ; p=0.342$ & 14.6 (2.29-93.1); $p=0.005^{*}$ \\
\hline No & Ref. & 1 & 1 \\
\hline \multicolumn{4}{|l|}{ Dominant artery } \\
\hline Right & Ref. & $5.67(0.91-35.4) ; p=0.063$ & $0.33(0.06-1.90) ; p=0.213$ \\
\hline Left & Ref. & $1.16(0.12-10.98) ; p=0.900$ & $0.12(0.01-1.16) ; p=0.067$ \\
\hline Both & Ref. & 1 & 1 \\
\hline \multicolumn{4}{|l|}{$M V D$} \\
\hline Yes & Ref. & $13.13(0.60-285.7) ; p=0.101$ & $0.95(0.04-20.9) ; p=0.975$ \\
\hline No & Ref. & 1 & 1 \\
\hline \multicolumn{4}{|c|}{$\begin{array}{l}\text { Significance levels: for unadjusted/univariate analysis }<0.25 \text {, for multivariate analysis }<0.05 \text {. } \\
\text { Multinomial logistic regression used. The reference category is: "best medical therapy". OR: odd ratio }(95 \% \text { confidence } \\
\text { interval). PCI: percutaneous coronary intervention, CABG: coronary artery bypass grafting, } \\
\text { NSTEMI: non-ST-elevation myocardial infarction, STEMI: ST-elevation myocardial infarction, LM: left main lesion, RCA: } \\
\text { right coronary arteries, LAD: left anterior descending artery, LCX: left circumflex artery, MVD: coronary microvascular disease, } \\
\text { *statistically significance value }\end{array}$} \\
\hline
\end{tabular}


deaths in men and women, respectively. ${ }^{13}$ This gender difference in the burden of CAD can be attributed to some variations in risk profile, pathophysiological mechanisms, and diagnosis of CAD between men and women. ${ }^{14}$ Indeed, atypical presentation of the CAD, which is mainly due to microvascular angina and more common in women, is associated with poorer outcomes. ${ }^{13}$ Hence this form of CAD warrants special medical care in affected women. ${ }^{13}$

Clinical manifestation of CAD varies with the culprit vessel. ${ }^{15}$ Akin to the present study's findings, previous studies have also reported the LAD artery as the most common culprit vessel. The involvement of LAD leads to worse outcomes. ${ }^{16}$ On the contrary, a study conducted by Aldosari et a ${ }^{17}$ reported LCX as the most common artery involved in CAD, followed by the LAD, the right circumflex artery, and LM on coronary angiography in the Saudi population. This discrepancy needs to be further evaluated in larger studies.

The incidence of CAD and its complications increases with advanced age. Cardiac interventions, such as CABG or PCI, are therefore increased in old age. Pacaric et $\mathrm{al}^{18}$ evaluated 47 participants aged $30-75$ years who underwent CABG and found that approximately half of the patients (49\%) were >60 years old and $55 \%$ of them were males. Smoking, an established risk factor for $\mathrm{CAD}$, also independently increases the risk of complications of cardiac surgery. The incidence of multiple comorbidities among the patients undergoing CABG has increased from that of previous years. ${ }^{19}$

Study limitations. The main limitation of the study was the relatively small sample. Despite the inclusion of patients from 3 facilities, our findings are not completely representative of the CAD population in the KSA. Variations in patients' profiles and physician practices can be observed across settings. Furthermore, some details were not available, including the severity of CAD lesions and the extent of CABG (double, triple, or quadruple bypass graft). Notwithstanding these limitations, this study provides crucial information on the risk profile and treatment choices for patients with CAD in the KSA. Furthermore, provided that only patients presenting to the hospital were included in the study, those in the population with very mild symptoms may have been missed out. Owing to this sampling bias, results from this study may not be extrapolated to the general population. Prospectively designed larger multicenter studies are required to better capture the epidemiology, treatment, and outcomes of the CAD in the country.

In conclusion, this study shows there exists a high prevalence of hypertension, diabetes, dyslipidemia, and history of smoking among patients with CAD in our settings. This important finding highlights the need to tackle these $\mathrm{CV}$ risk factors for the primary prevention of CAD. Most patients who presented themselves with CAD in our settings were treated conservatively, and more than half of them were managed with BMT alone. Patients were less commonly managed with PCI or CABG. Patients' age and the anatomical localization of coronary atherosclerotic lesions were the main determinants of treatment with CABG or PCI. To the best of our knowledge, there is no study that delineates the association between patient factors and modality of CAD treatment-CABG or PCI, specific to the Saudi population. The strength of these associations is yet to be confirmed with further studies. Although optimal medical therapy is pivotal in all patients, there is a need to scale up the use of PCI to meet international recommendations and improve survival.

Acknowledgment. We are grateful to the Deanship of Scientific Research at Prince Sattam Bin Abdulaziz University for their support of this research project under the research project \#2020/03/16884.

\section{References}

1. Global burden of 369 diseases and injuries in 204 countries and territories, 1990-2019: a systematic analysis for the Global Burden of Disease Study 2019. Lancet (London, England) 2020; 396: 1204-1222.

2. Saeed AA, Al-Hamdan NA, Bahnassy AA, Abdalla AM, Abbas MA, Abuzaid LZ. Prevalence, awareness, treatment, and control of hypertension among Saudi adult population: a national survey. Int J Hypertens 2011; 2011: 174135.

3. Al-Nozha MM, Arafah MR, Al-Maatouq MA, Khalil MZ, Khan NB, Al-Marzouki K, et al. Hyperlipidemia in Saudi Arabia. Saudi Med J 2008; 29: 282-287.

4. Al-Nozha MM, Al-Maatouq MA, Al-Mazrou YY, Al-Harthi SS, Arafah MR, Khalil MZ, et al. Diabetes mellitus in Saudi Arabia. Saudi Med J 2004; 25: 1603-1610.

5. Schwartz L. Therapeutic options in coronary artery disease: focusing on the guidelines. Can J Cardiol 2009; 25: 19-24.

6. Windecker S, Stortecky S, Stefanini GG, DaCosta BR, Rutjes AW, Di Nisio M, et al. Revascularisation versus medical treatment in patients with stable coronary artery disease: network meta-analysis. BMJ 2014; 48: g3859.

7. Melly L, Torregrossa G, Lee T, Jansens JL, Puskas JD. Fifty years of coronary artery bypass grafting. $J$ Thorac Dis 2018; 10 : 1969-1967.

8. Ezhumalai B, Jayaraman B. Angiographic prevalence and pattern of coronary artery disease in women. Indian Heart $J$ 2014; 66: 422-426.

9. Task Force Members, Montalescot G, Sechtem U, Achenbach S, Andreotti F, Arden C, et al. 2013 ESC guidelines on the management of stable coronary artery disease: the Task Force on the management of stable coronary artery disease of the European Society of Cardiology. Eur Heart J 2013; 34: 2949-3003. 
10. Wakabayashi I. Gender differences in cardiovascular risk factors in patients with coronary artery disease and those with type 2 diabetes. J Thorac Dis 2017; 9: E503-E506.

11. Sakboonyarat B, Rangsin R. Prevalence and associated factors of ischemic heart disease (IHD) among patients with diabetes mellitus: a nation-wide, cross-sectional survey. BMC Cardiovasc Disord 2018; 18: 151.

12. Park JJ, Park SJ, Choi DJ. Microvascular angina: angina that predominantly affects women. Korean J Intern Med 2015; 30 : 140-147.

13. Tomaszewski M, Topyła W, Kijewski BG, Miotła P, Waciński P. Does gender influence the outcome of ischemic heart disease? Prz Menopauzalny 2019; 18: 51-56.

14. Taqueti VR. Sex Differences in the coronary system. Adv Exp Med Biol 2018; 1065: 257-278.

15. Entezarjou A, Mohammad MA, Andell P, Koul S. Culprit vessel: impact on short-term and long-term prognosis in patients with ST-elevation myocardial infarction. Open Heart 2018; 5: e000852.
16. Ghanim D, Kusniec F, Kinany W, Qarawani D, Meerkin D, Taha K, et al. Left circumflex coronary artery as the culprit vessel in st-segment-elevation myocardial infarction. Tex Heart Inst J 2017; 44: 320-325.

17. Aldosari KH, Alkhathlan KM, Al-Ghamdi S, Abdelhamid Elshaer FE, Karrar MH, Aldawsari AM. Pattern of coronary arterial lesions amongst Saudi Arabians: a cross-sectional coronary fluoroscopic angiography study. Pan Afr Med J 2020; 36: 21.

18. Pačarić S, Turk T, Erić I, Orkić Ž, Petek Erić A, Milostić-Srb A, et al. Assessment of the quality of life in patients before and after Coronary Artery Bypass Grafting (CABG): a prospective study. Int J Environ Res Public Health 2020; 17: 1417.

19. Dadkhah-Tirani H, Hasandokht T, Agostoni P, Salari A, Shad B, Soltanipour S. Comparison of cardiovascular risk factors among coronary artery bypass graft patients in 2010 and 2016: A single-center study in Guilan province, Iran. ARYA Atheroscler 2018; 14: 205-211. 